\title{
Cell Type-specific Knockout with Gli1-mediated Cre Recombination in the Developing Cerebellum
}

\author{
Jung-Mi Choi $^{1 \dagger}$, Rakshya Acharya ${ }^{1,2 \dagger}$, Subash Marasini ${ }^{3}$, Bashyal Narayan ${ }^{1,2}$, Kwang-Wook Lee $^{1}$, \\ Woo Sup Hwang ${ }^{1}$, Da-Young Chang ${ }^{3}$, Sung-Soo Kim ${ }^{1,2 *}$ and Haeyoung Suh-Kim ${ }^{1,2,3 *}$ \\ ${ }^{1}$ Department of Anatomy, Ajou University School of Medicine, Suwon 16499, ${ }^{2}$ Department of Biomedical Sciences, Graduate \\ School, Ajou University School of Medicine, Suwon 16499, ${ }^{3}$ Research Center, CelleBrain Ltd., Jeonju 54871, Korea
}

\begin{abstract}
The inducible $C r e-l o x P$ system provides a useful tool for inducing the selective deletion of genes that are essential for proper development and enables the study of gene functions in properly developed animals. Here, we show that inducible Cre-lox $P$ driven by the Gli1-promoter can induce cell-type-specific deletion of target genes in cerebellar cortical neurons. We used reporter mice containing the YFP (yellow fluorescence protein) gene at the Gt(ROSA)26Sor locus with a lox P-flanked transcriptional stop sequence, in which successful Cre-mediated excision of the stop sequence is indicated by YFP expression in Cre-expressing cells. Administration of tamoxifen during early postnatal days (P4 7) induces Cre-dependent excision of stop sequences and allows YFP expression in proliferating neuronal progenitor cells in the external granule layer and Bergmann glia in the Purkinje cell layer. A substantial number of YFP-positive progenitor cells in the external granule layer migrated to the internal granule cell layer and became granule cell neurons. By comparison, injection of tamoxifen during late postnatal days (P19 22) induces YFP expression only in Bergmann glia, and most granule cell neurons were devoid of YFP expression. The results indicate that the Glil promoter is temporarily active in progenitor cells in the external granule layer during the early postnatal period but constitutively active in Bergmann glia. We propose that the Gli1mediated CreER system can be applied for the conditional deletion of genes of interest from cerebellar granule cell neurons and/or Bergmann glia.
\end{abstract}

Key words: Cerebellum, Cre recombinase, Tamoxifen, Gli1, Bergmann glia, Granule cell neuron

\section{INTRODUCTION}

The Cre-loxP recombination system has been widely used for studying gene functions in animals by allowing region-specific knockout of target genes through site-specific expression of Cre. Inducible Cre such as CreER provides more specific control of spatiotemporal deletion or lineage labeling through timed administration of synthetic estrogen receptor (ER) ligands such as

Submitted June 9,2021, Revised June 29, 2021,

Accepted June 29,2021

* To whom correspondence should be addressed.

Sung-Soo Kim, TEL: 82-31-219-5036, FAX: 82-31-219-5034

e-mail:kimdmg@ajou.ac.kr

Haeyoung Suh-Kim, TEL: 82-31-219-5036, FAX: 82-31-219-5039

e-mail:hysuh@ajou.ac.kr

${ }^{\dagger}$ These authors contributed equally to this work. tamoxifen (TAM) or 4-hydroxytamoxifen (4-OHT) [1, 2]. Embryonic administration of tamoxifen rapidly induces abortion in pregnant mouse mothers and severely perturbs embryonic development, rendering the inducible Cre system inapplicable to the study of developmentally regulated genes in embryos [3]. In contrast, tamoxifen injection after birth is less harmful and relatively tolerable in neonates, suggesting that inducible Cre-loxP may provide genetic tools for the study of postnatal development [4]. While most major structures in the central nervous system develop before birth, cerebellar architecture develops actively during the first three weeks after birth [5]. During this period, neuronal progenitor cells proliferate, migrate, and terminally differentiate into the cerebellar cortex. Postnatal injection of tamoxifen to neonates may enable studies on developmentally regulated gene functions in mitotic and/or postmitotic cells in the cerebellum.

The cerebellar cortex consists of three distinct layers: the mo-
Copyright ( $)$ Experimental Neurobiology 2021. www.enjournal.org
This is an Open Access article distributed under the terms of the Creative Commons Attribution Non-Commercial License (http://creativecommons.org/licenses/by-nc/4.0) which permits unrestricted non-commercial use, distribution, and reproduction in any medium, provided the original work is properly cited. 
lecular layer (ML), Purkinje cell layer (PCL), and granule cell layer (GCL). The somata of Purkinje cells (PCs) and Bergmann glia (BGs) are arranged in a single PCL layer. PCs are the only output neurons of the cerebellar cortex, and each sends a single, long axon to the deep cerebellar nuclei (DCN). ML contains inhibitory interneurons, parallel fibers of GCNs, $\mathrm{PC}$ dendrites, and $\mathrm{BG}$ radial fibers. The complex dendrites of PCs in the ML receive presynaptic inputs from parallel fibers (PFs) originating in GCNs in the GCL and climbing fibers (CFs) projecting from the inferior olivary nucleus [6]. This trilaminar architecture of the cerebellar cortex developed perinatally. During the late embryonic period (E17.5) and postnatal development, cerebellar granule cell progenitors (GCPs) rapidly proliferate in the external granule layer (EGL), radially migrate to the internal GCL, and eventually differentiate into GCNs. Sonic hedgehog (Shh) plays a key role in the proliferation of GCPs during cerebellar morphogenesis and histogenesis, and deletion of Shh induces hypoplasia of the cerebellar cortex [79]. Shh secreted by PC $[9,10]$ activates the Glil promoter in GCPs and BGs [7, 11-13]. Thus, Gli1 ${ }^{\text {CreERT2 }}$ mice with CreERT2 knocked into the Glil locus are widely used as a readout of Shh-positive signaling to study the contribution of Shh transcriptional activator function during postnatal development [14-16]. Recently, several proteins from the synaptic compartment have been shown to have a longer half-life than those from the cytoplasmic compartment [17]. The long half-life of proteins makes it difficult to sufficiently clear these gene products from post-mitotic neurons even after the mRNA and protein are no longer synthesized after genetic recombination. Glit ${ }^{\text {CreERT2 }}$ mice may provide a useful system for knocking out these genes in post-mitotic neurons by allowing the dilution of proteins in dividing progenitor cells.

In this study, we investigated whether Gli1 ${ }^{\text {CreERT2 }}$ could provide an appropriate system to selectively knockout genes in proliferating GCPs and whether the knockout effect was maintained in post-

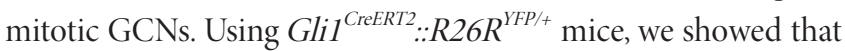
tamoxifen administration in the early and late postnatal period leads to cell-type specific knockout of the target genes in GCNs and/or BGs. Tamoxifen administration at P4 7 induces recombination in BGs and proliferating GCPs, leading to GCN knockout. Thus, tamoxifen administration at P19-22 induces knockout only in BGs. These results suggest that the Gli1 promoter leads to spatial deletion in GCPs and BGs, and timed administration of tamoxifen further specifies temporal deletion in GCNs and BGs. We also propose that Gli1 ${ }^{\text {CreERT2 }}$ mediated recombination in proliferating GCPs may aid in the study of synaptic proteins with an extremely long half-life.

\section{MATERIALS AND METHODS}

\section{Mouse genetics}

Gli $1^{\text {tm3(cre/ERT2)Alj }} / \mathrm{J}$ (referred to as Gli $\left.1^{\text {CreERT2 }}, \# 007913\right)$ and B6.129X1-Gt(ROSA)26Sor ${ }^{\text {tml(EYFP)Cos }}$ (referred to as R26R-YFP, \#006148) have been previously described [14, 18]. Gli I ${ }^{\text {CrefRT2 }}$ mice were crossed with R26R-YFP mice to generate heterozygotes for each allele and used to determine Cre-mediated recombination. $G l i 1^{+1+}:: R 26 R^{Y F P /+}$ was used as a negative control to demonstrate the specificity of tamoxifen (TAM) administration. Offspring were genotyped by polymerase chain reaction (PCR) with genomic DNA (gDNA) as previously described [19] using the primers shown in Table 1.

TAM (Sigma-Aldrich) was dissolved in corn oil to a final concentration of $10 \mathrm{mg} / \mathrm{ml}$. To activate Cre recombinase, mice were force-fed TAM ( $50 \mu \mathrm{g} / \mathrm{g}$ weight/ day) by oral pipet-feeding at the indicated time points and housed until euthanized.

All experimental procedures were approved by Ajou University Medical Center-Institutional Animal Care and Use Committee (AUMC-IACUC, Suwon, South Korea).

\section{Immunofluorescence analysis}

Immunohistochemical analyses were performed as described previously [20]. Briefly, mice were deeply anesthetized with 2,2,2

Table 1. The primer sequences for PCR reaction

\begin{tabular}{|c|c|c|}
\hline Target & Primer sequence & PCR product \\
\hline \multicolumn{3}{|l|}{$R 26 R$} \\
\hline Forward & 5'AAA GTC GCT CTG AGT TGT TAT-3' & WT: $600 \mathrm{bp}(\mathrm{F}+\mathrm{R} 1)$ \\
\hline Reverse 1 & 5'-GGA GCG GGA GAA ATG GAT ATG-3' & Knock-in: 325 bp $(F+R 2)$ \\
\hline Reverse 2 & 5'-GCG AAG AGT TTG TCC TCA ACC-3' & \\
\hline \multicolumn{3}{|c|}{$\triangle R 26 R-Y F P($ recombined $)$} \\
\hline Forward & 5'-GCG AAG AGT TTG TCC TCA ACC-3' & $750 \mathrm{bp}$ \\
\hline Reverse & 5'ATG GCG GAC TTG AAG AAG TCG TG-3' & \\
\hline \multicolumn{3}{|l|}{ Gli1-CreERT2 } \\
\hline Forward & 5'-GCA TTA CCG GTC GAT GCA ACG AGT GAT GAG-3' & $404 \mathrm{bp}$ \\
\hline Reverse & 5'-GAG TAG ACG AAC CTG GTC GAA ATC AGT GCG-3' & \\
\hline
\end{tabular}


tribromoethanol (200 mg/kg, i.p., Sigma-Aldrich), and then perfused transcardially with $10 \%$ neutral buffered formalin (BBC Biochemical). The brain was extracted, post-fixed in $10 \%$ neutral buffered formalin overnight at $4{ }^{\circ} \mathrm{C}$, and cryoprotected in $30 \%$ sucrose. The brain was sagitally divided into two halves, embedded in OCT compound (Tissue-Tek, Sakura Finetek), and sectioned into $30 \mu \mathrm{m}$-thick frozen sections using a Leica cryostat (Leica). The cryosections were air-dried, and the residual OCT compound was washed in PBS with $0.1 \%$ (v/v) Triton X-100 (Sigma-Aldrich) (PBS-T). After incubation in blocking solution [10\% ( vol/vol) normal goat serum (Gibco), 1\% bovine serum albumin (BSA; SigmaAldrich) in PBS-T] for $1 \mathrm{~h}$ at room temperature, the sections were incubated with primary antibodies overnight at $4^{\circ} \mathrm{C}$. The antibodies used in this study were as follows: anti-GFP (1:500, Abcam, \#ab13970), anti-Pcp2 (1:500, Santa Cruz, \#sc-137064), anti-GFAP (1:200, Dako, \#Z0334), anti-S100ß (1:500, Abcam, \#ab41548), antiNeuN (1:500, EMD Millipore, \#MAB377), and anti-parvalbumin (PV; 1:500; Swant, \#PV25). After unbound antibodies were washed with PBS-T, the sections were incubated with secondary antibodies conjugated with Alexa Fluor 405, 488, or 568 (1:500, Invitrogen). If necessary, nuclear counterstaining was performed using bisbenzamide (1:50,000, Hoechst 33258; Invitrogen). All fluorescence images were acquired using a Zeiss LSM710 confocal laser scanning microscope (Carl Zeiss) or Zeiss Axio Scan Z1 slide scanner (Carl Zeiss) at the Three-Dimensional Immune System Imaging Core Facility of Ajou University.

\section{Quantitative analysis}

Confocal images of cerebellar sagittal sections were analyzed using ZEN software (Blue Edition, Zeiss). The specificity of Gli1CreER2 were expressed as the ratio of $\mathrm{NeuN}^{+}$GCNs among $\mathrm{YFP}^{+}$ cells in GCL or the ratio of S100 $\beta^{+}$BGs in PCL/ML. The coverage was shown as the ratio of $\mathrm{YFP}^{+}$cells in $\mathrm{NeuN}^{+}$GCNs or in $\mathrm{S} 100 \beta^{+}$ cells as previously defined [21].

\section{RESULTS}

\section{Cerebellum specific Cre-mediated recombination driven by Gli1 promoter}

To assess the Gli1 promoter-mediated expression of the Cre enzyme, Gli1 ${ }^{\mathrm{CreER} 2 /+}:: R 26 R^{Y F P /+}$ mice were obtained by breeding Gli1 ${ }^{\mathrm{CreER} 2 /+}$ mice carrying the CreERT2 gene under the Gli1 promoter with $R 26 R-Y F P$ reporter mice that harbored a floxed stop cassette upstream of the enhanced yellow fluorescent protein gene (YFP) at the ubiquitously expressed ROSA locus [18]. Genotyping was performed by PCR using gDNA obtained from the tail biopsy or cerebellum. The wildtype and floxed al- leles were detected as $600 \mathrm{bp}$ and $325 \mathrm{bp}$ fragments, respectively (Table 1).

The TAM-activated Cre enzyme excised the stop cassette flanked by two loxP sites and permitted YFP expression in Gli1-expressing cells. Cre-mediated recombination was validated using a $750 \mathrm{bp}$ PCR product with gDNA isolated from the cerebellum. Such PCR products were not detected in the cerebral cortex, suggesting that the Gli1 promoter is active only in the cerebellum (Fig. 1C). Consistently, in the sagittal sections of Gli1 ${ }^{\mathrm{CreERT} 2 /+}:: R 26 R^{Y F P /+}$ brain, YFP expression was detected only in the cerebellar cortex, including the ML, PCL, and GCL, but not in the white matter or other brain regions (Fig. 1D). The results indicated that administration of TAM during early postnatal periods induces recombination in the cerebellar cortex.

\section{Gli1 active cells in early postnatal cerebellum}

The proliferation of GCPs reaches its peak at P4-8 in response to Shh produced by PCs [22]. To determine the cell types responsive to Shh in early postnatal period, we administered TAM at the peak time of the GCP proliferating period (P4 7) and sacrificed the mice at P9 (Fig. 2A). YFP expression was detected in proliferating GPCs in EGL. YFP+ cells migrated to the GCL and became fully differentiated $\mathrm{NeuN}^{+}$GCNs. YFP expression was also detected in radially extending fibers in the $\mathrm{ML}$ and soma of $\mathrm{GFAP}^{+} \mathrm{BGs}$ in the PCL (Fig. 2C and 2D). YFP was not expressed in Pcp2 ${ }^{+}$PCs in the PCL (asterisks in Fig. 2D") or parvalbumin (PV) ${ }^{+}$including GABAergic inerneurons and PCs (Fig. 3). These results suggest that the administration of tamoxifen during early postnatal days induces expression of Cre recombinase in Gli1-expression GCPs and BGs, but not in other types of cells in the developing cerebellum.

\section{Cerebellar granule cells- and Bergmann glial cells-specific expression of Cre recombinase activity}

To determine the duration of Shh-responsiveness, we administered TAM at two distinct time points: during P4 7, when GCPs in the EGL migrated to the internal GCL and during P19 22 when EGL no longer existed [23]. YFP expression that was induced by TAM at P4 7 in GCPs and BGs was maintained in GCNs and BGs in the mature brain at P25 (Fig. 4B and 4D). In contrast, TAM administration at P19 22 induced YFP expression only in BGs, but not in GCs (Fig. 4C and 4E). For quantification, we performed additional staining with anti-S100 $\beta$. GFAP and S100 $\beta$ revealed the same cell population with distinctive immunoreactivity: S100 $\beta$ in the somata and proximal processes of BGs and GFAP in the arborized glial fibers. TAM injection at P4 7 induced $\mathrm{YFP}^{+}$ expression in $15.1 \pm 1.4 \%$ of $\mathrm{NeuN}^{+} \mathrm{GCNs}$ and $18.0 \pm 1.8 \%$ of BGs (Fig. 4F and 4G). The specificity of YFP expression was high thus 
A

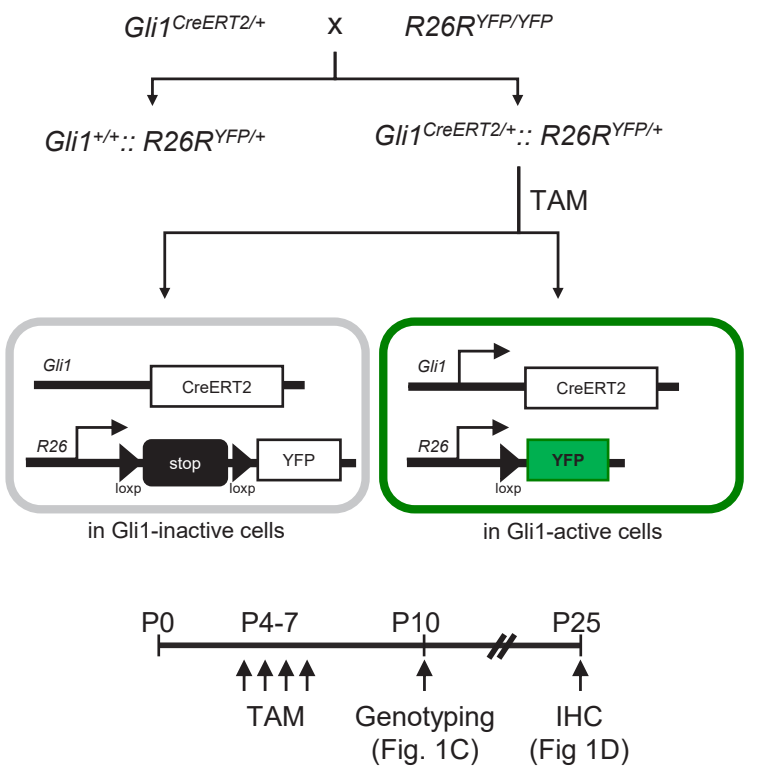

C
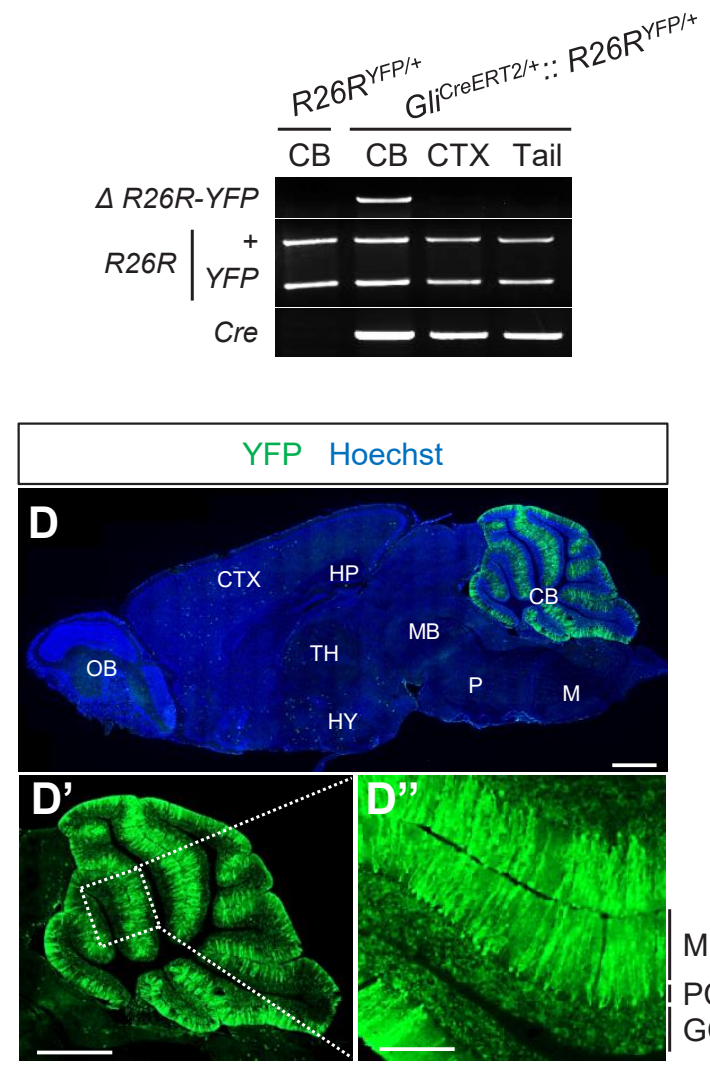

ML PCL GCL

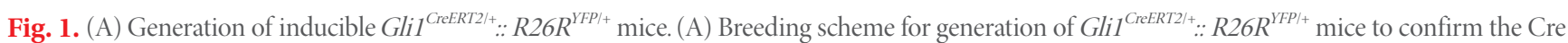
activity according to tamoxifen induction. (B) Schematic experimental design for the tamoxifen injection time points used in $G l i 1^{C r e E R T 2 /+}:: R_{26} R^{Y F P /+}$ mice. Pups were orally injected TAM on P4 7. Pups were sacrificed to collect the brain and tail samples at different indicated time points. (C) Representative genotyping results of offspring from $R 26 R^{Y F P Y Y P}$ reporter and $G l i 1^{\text {CreERT2/+ }}$ crosses. Genomic DNA (gDNA) from brain regions (cerebellum and cortex) and tail were examined by PCR for recombination of floxed alleles in $G l i 1^{\text {CreERT2/+ }}:: R 26 R^{Y F P /+}$ mice. Recombinant $\triangle R 26 R-Y F P$, which indicates the transgene of cre-mediated recombination driven by Glil activation was observed in cerebellum but not in cerebral cortex and tail of Glil $^{\mathrm{CreFRT2/+}}:$. $R 26 R^{Y F P /+}$ mice. Mice not expressing Cre from parallel breeding were taken as control (lane 1). (D D") Sagittal whole-brain images from tamoxifen injected Gli1 ${ }^{\mathrm{CrERT} 2 /+}:: R^{2} 2 R^{Y F P /+}$ mouse at P25, indicating that Cre-recombinase is selectively expressed in the cerebellum. Green fluorescence, YFP signal indicates Cre-mediated recombination. Counterstaining was performed using Bisbenzimide (Hoechst 33342) dye. (D') YFP signal was dominantly

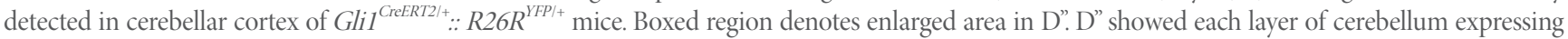
YFP signal. P, postnatal day; TAM, tamoxifen; IHC, Immunohistochemistry; CB, cerebellum; CTX, cerebral cortex; OB, Olfactory bulb; HP, Hippocampus; TH, thalamus; HY, Hypothalamus; MB, Midbrain; P, Pons; M, Medulla; ML, Molecular layer; PCL, Purkinje cell layer; GCL, Granule cell layer. Scale bars $=1 \mathrm{~mm}$ in D and D', $0.2 \mathrm{~mm}$ in D".

most $\mathrm{YFP}^{+}$cells in GCL or PCL were NeuN ${ }^{+}$GCN $(98.6 \pm 0.46 \%)$ or BGs (100\%), respectively. When TAM was injected at P19 22, the $\mathrm{YFP}^{+}$expression was found in $45.8 \pm 2.9 \%$ to BGs in PCL with $99.5 \pm 0.47 \%$ specificity. None of GCNs were co-localized with YFP expression. The results indicated that Shh signaling is temporarily active in the proliferation of GCP and BG during the early postnatal period and constitutively active only in BGs.

\section{DISCUSSION}

This study showed that timed postnatal administration of TAM differentially regulates cell-type-specific excision of floxed genes in the developing cerebellar cortex. TAM administration at early postnatal days (P4 7), when the GCP proliferation peaks in the EGL, can induce the expression of Glil-mediated Cre recombinase in GCPs of EGL and BGs in PCL. Thus, tamoxifen administration during postnatal days (P19 22), when EGL is almost depleted, leads to the expression of YFP only in BGs. Our results are consistent with the previous finding that Glil expression is restricted to proliferating GCPs and BGs in the developing cerebellar cortex in response to Purkinje-derived Shh in postnatal stages through adulthood $[10,13,24,25]$. Importantly, YFP ${ }^{+}$GCPs in the EGL inwardly migrate to and differentiate $\mathrm{NeuN}^{+}$GCNs in the GCL, where they remain as $\mathrm{YFP}^{+}$GCNs to adulthood (Fig. 2). 

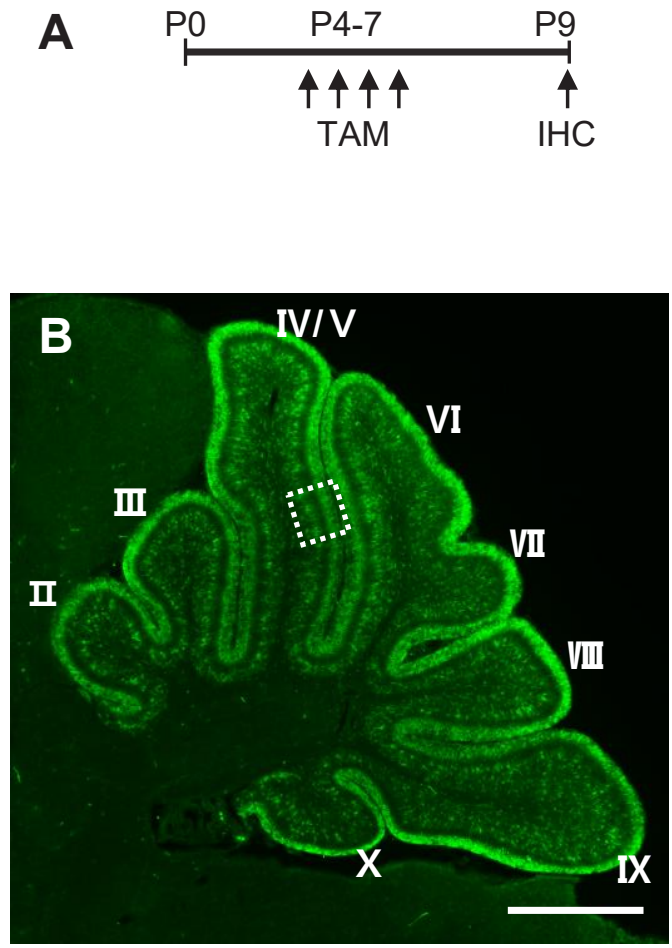
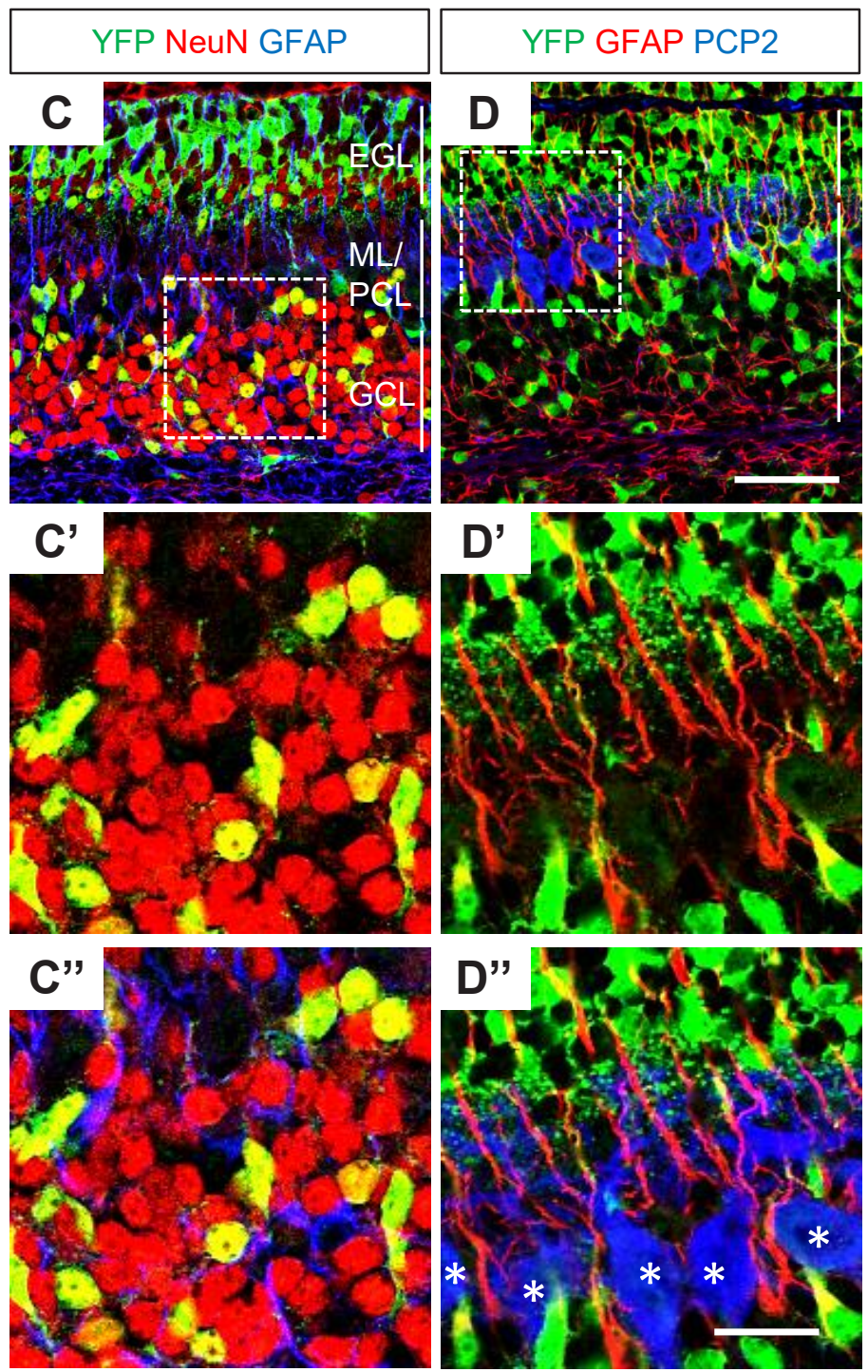

Fig. 2. Glil-mediated Cre recombination in proliferation EGL, ML, and GCL. (A) Schematic experimental design for the tamoxifen injection time points used in Gli1 ${ }^{\mathrm{CreERT2/+}}:: R 26 R^{Y F P /+}$ mice. Pups were orally injected TAM at P4-7 and sacrificed at P9 to analyze the YFP-expressing cells. (B) YFP la-

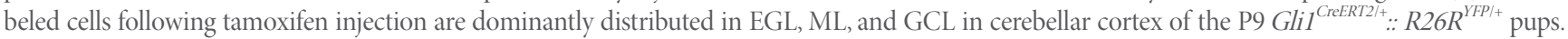
The lobules of vermis are identified by Roman numerals (II-X). Boxed region denotes enlarged area in C and D. (C, D) Triple staining for YFP, NeuN (a marker for GCs), PCP2 (a marker of PCs) and/or GFAP (a marker of BGs) of sagittal cerebellum of Gli1 $_{\text {CreERT2/+.: }}^{\text {R26R }}{ }^{Y F P /+}$ showed that YFP signal was colocalized with NeuN ${ }^{+}$proliferating GCPs in EGL (C), differentiated GCNs in ML (C' C"), GFAP ${ }^{+}$BGs in ML/PCL (D D"), but not in PCP2 ${ }^{+}$PCs (D and D"). Boxed region in C and D denotes enlarged area in C, C", D' and D". In D". Asterisks indicated soma of PCs. P, postnatal day; TAM, tamoxifen; IHC, Immunohistochemistry; EGL, external granule cell layer; ML, Molecular layer; PCL, Purkinje cell layer; GCL, Granule cell layer. Scale bars=500 $\mu$ m in $\mathrm{B}, 50 \mu \mathrm{m}$ in $\mathrm{C}$ and $\mathrm{D}, 20 \mu \mathrm{m}$ in $\mathrm{C}^{\prime} \sim \mathrm{D}^{\prime \prime}$.

As mentioned earlier, proteins with long half-lives in the synaptic compartment [17] may remain in postmitotic GCNs even after the mRNA and protein are no longer synthesized. Gli1-CreERT2 may be advantageous for inducing genuine deficiency of the gene products by targeting GCPs, and the protein products are diluted during cell division.

\section{Shh-Gli1 signaling in the developing cerebellum}

All cerebellar neurons are generated from progenitors in two distinct germinative centers in the hindbrain: the rhombic lip and the ventricular zone $[5,26]$. The progenitor cells in the rhombic lip express Math-1 (mouse homolog-1 of Drosophila Atonal) and generate glutamatergic neurons, including projection neurons in deep cerebellar nuclei, unipolar brush cells, and GCNs in the GCL 


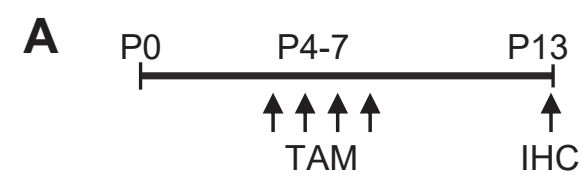

B

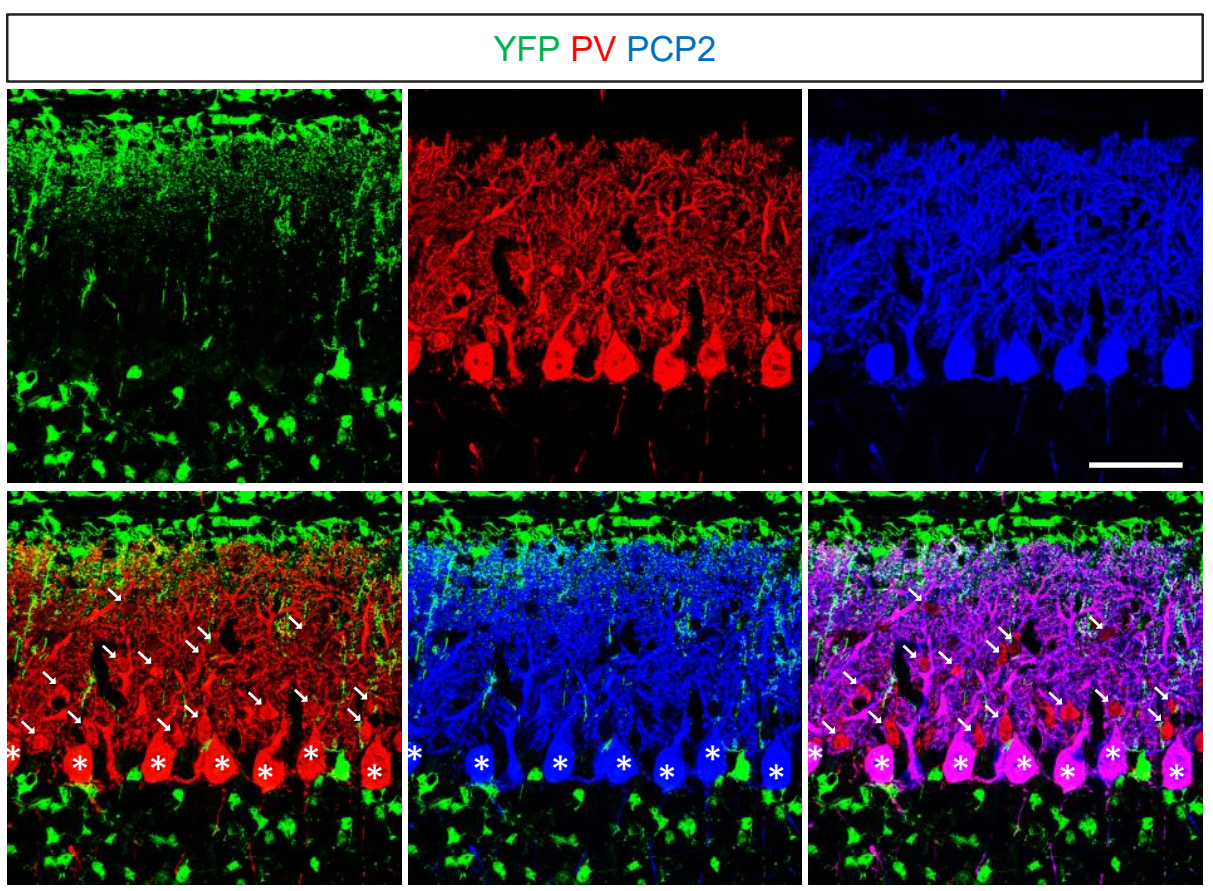

Fig. 3. Glil-mediated Cre recombination in ML. (A) Schematic representation of the experimental design. Pups were injected with TAM at P4 7. Brain sections were prepared for analysis at the P13. (B) YFP signal was not found in either PV ${ }^{+}$neurons (PCs and GABAergic interneurons in ML) and PCP2 ${ }^{+}$ PCs. Arrows indicate $\mathrm{PV}^{+}$inhibitory interneurons (basket and stellate cells) in ML, and asterisks indicate soma of PCs. Scale bar: $50 \mu \mathrm{m}$.

$[27,28]$. The progenitor cells in the ventricular zone generate all GABAergic phenotypes, including PCs, nucleo-olivary projection neurons, and all inhibitory interneurons, astrocytes, and oligodendrocytes in the white matter $[29,30]$. Shh produced by PCs acts as a mitogen on progenitor cells originating from the rhombic lip and ventricular zone [24,31] affects Bergmann glial differentiation [12]. Based on Glil expression, a high level of positive Shh signaling is restricted to the proliferating GCPs and BGs in developing cerebellar cortex [13,24]. Only BGs have been shown to be capable of responding to PC-derived Shh signals in postnatal stages through adulthood [25]. Consistently, TAM activates Gli1-CreERT2 in Shh-responsive GCPs in the EGL and BGs in the PCL (Fig. 1 and 2). Shh is also known to exert a proliferative function on neural stem cell-like progenitors in the white matter around P1 2 [9, 15, 32]. These progenitor cells $\left(\mathrm{Tnc}^{+}, \mathrm{CD} 133^{+}\right)$give rise to GABAergic progenitor cells $\left(\mathrm{Ptfla}^{+}\right)$and astrocyte precursors $\left(\mathrm{Tnc}^{+}, \mathrm{CD} 15^{+}\right)$, which eventually differentiate into interneurons, oligodendrocytes, astrocytes, and BGs $[9,30,33]$. Thus, TAM administration at P1-3 activates Gli1-CreERT2 in these progenitor cells, leading to the expression of reporter genes in interneurons and astrocytes [15]. However, we did not detect YFP expression in GABAergic neurons when TAM was administered at P4 7 (Fig. 3). Our results are consistent with the notion that the entire repertoire of GABAergic interneurons in the cerebellar cortex is generated before P7 with a peak around P5 [29, 30, 34]. Importantly, timed administration is critical to conditionally knockout the target genes in glutamatergic GCNs without affecting gene expression in GABAergic interneurons.

\section{Cre-mediated knockout for the study of cerebellar cortex}

Several Cre lines have been used to knock out genes in specific cell types in the cerebellum (Table 2): GABAa6-Cre and Math1Cre for deletion in GCNs [35-38], and Pcp2/L7-Cre and ShhCre for deletion in PCs [15, 39-41]. While GABAa6-Cre is useful 
A
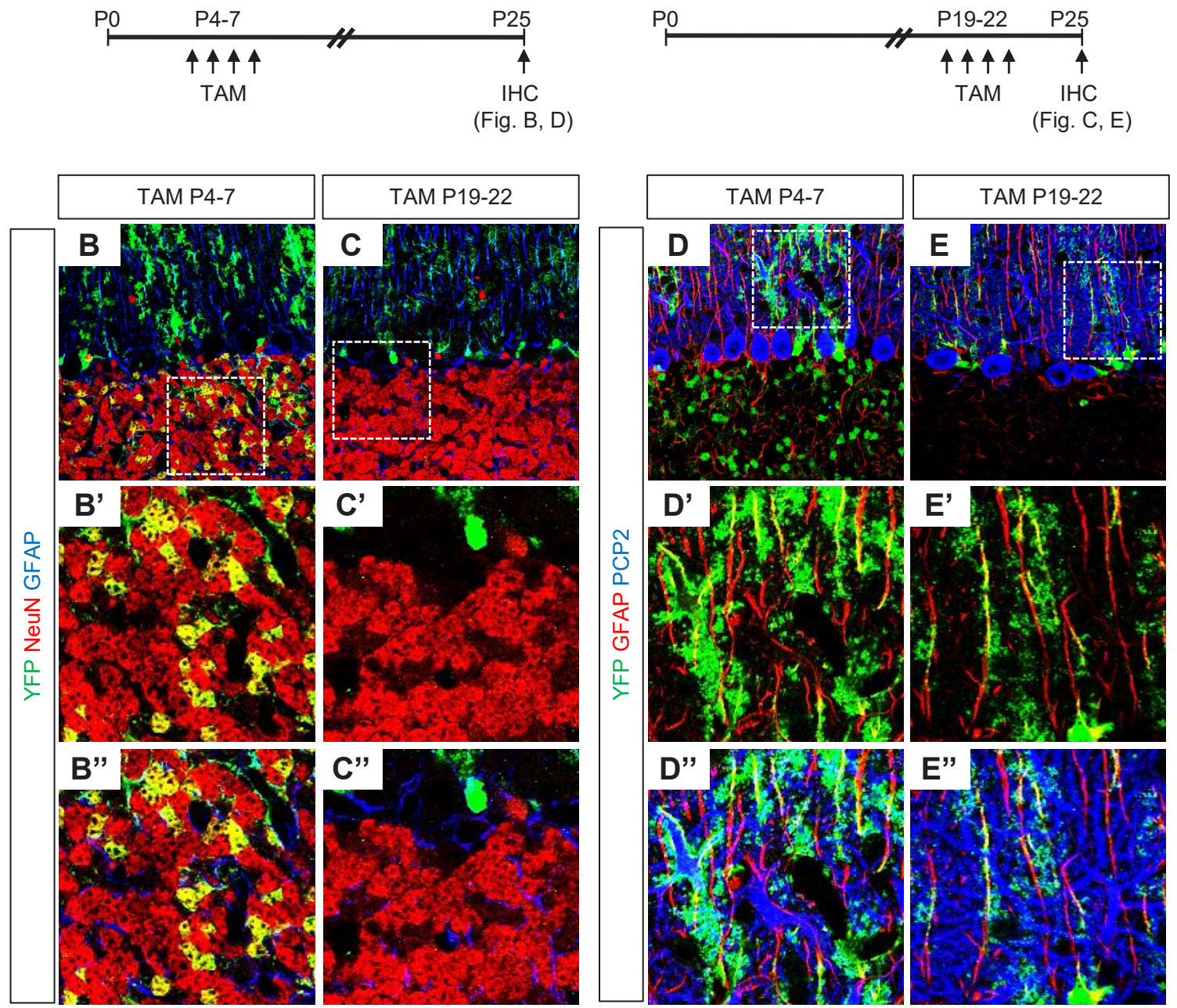

$\mathbf{F}$

G
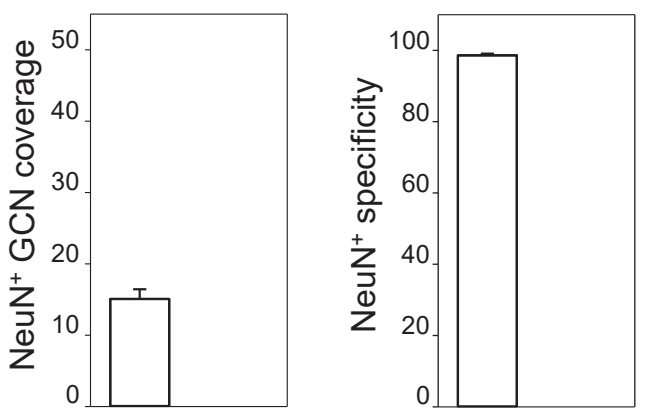

TAM P4-7 P19-22

TAM P4-7 P19-22
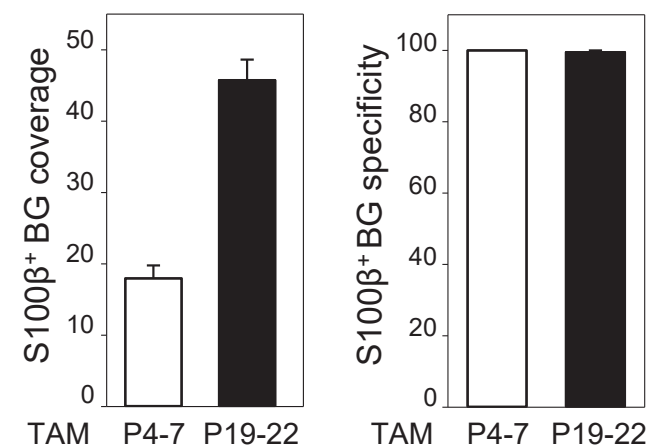

Fig. 4. Glil-mediated Cre recombination in GCNs and BGs. (A) Schematic experimental design for the tamoxifen injection time points used in Gli1 ${ }^{\text {Cre }}$ ${ }^{E R T 2 /+}:: R 26 R^{Y F P /+}$ mice. Pups were orally injected with TAM at P4 7 (left, for B and D) or P19 22 (right, for C, E), and sacrificed at P25 to analyze the YFPexpressing cells. (B E) Tamoxifen injection at different time point led to YFP labeled cells that were cerebellar cell-type and specifically regulated Cremediated recombination in P25 Gli1 ${ }^{C r e E R T 2 /+}:: R 26 R^{Y F P /+}$ cerebellum. Similar to P9 cerebellum, injection of TAM at P4 7 allowed YFP expression which was induced in NeuN ${ }^{+}$GCNs (Fig. B B"), GFAP ${ }^{+}$BGs (D D"), but not in PCP2 $2^{+}$PCs (D), whereas late administration of TAM at P19 22 led to detec-

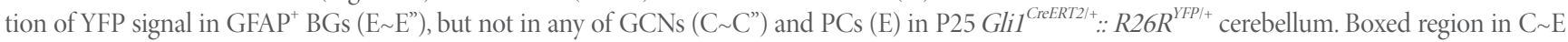
denotes enlarged area in C' $\sim E^{\prime \prime}$. Scale bars $=50 \mu \mathrm{m}$ in B E, $20 \mu \mathrm{m}$ in B' $\sim E^{\prime \prime}$. (F G) Colocalization of YFP ${ }^{+}$cells were assessed with confocal images from $4 \sim 6$ from sagittal sections of 2 3 animals per group as mentioned in the Methods. The specificity and coverage of YFP expression in NeuN ${ }^{+} \mathrm{GCNs}$ and in $S 100 \beta^{+}$are shown means \pm SEM. 
Table 2. Mouse lines expressing Cre recombinase in the cerebellum

\begin{tabular}{|c|c|c|c|c|c|c|}
\hline Mouse line & TAM @ & GCN & BG & PC & IN & References \\
\hline \multirow[t]{3}{*}{ Gli1-CreERT2 } & $\mathrm{P} 1 \sim 3$ & $\mathrm{~V}$ & $\mathrm{~V}^{*}$ & & $\mathrm{SC}, \mathrm{BC}$ & {$[15]$} \\
\hline & P4 7 & $\mathrm{V}$ & $\mathrm{V}$ & & & This study \\
\hline & Adult & & $\mathrm{V}$ & & & This study, [16] \\
\hline \multirow[t]{2}{*}{ NSE-CreERT2 } & $\mathrm{P} 1 \sim 3$ & V & & & & {$[42]$} \\
\hline & Adult & $\mathrm{V}$ & & & & {$[42]$} \\
\hline Math1-CreER & $\mathrm{P} 1 \sim 3$ & $\mathrm{~V}$ & & & $\mathrm{UBC}$ & {$[15]$} \\
\hline \multirow[t]{2}{*}{ GFAP-CreER } & P4 7 & & $\mathrm{V}^{*}$ & & & {$[45]$} \\
\hline & Adult & & $\mathrm{V}$ & & & {$[43]$} \\
\hline \multirow[t]{3}{*}{ GLAST-CreER } & $\mathrm{P} 1 \sim 3$ & & $\mathrm{~V}^{*}$ & & $\mathrm{SC}, \mathrm{BC}, \mathrm{UBC}$ & {$[44]$} \\
\hline & P4 7 & & $\mathrm{V}^{*}$ & & & {$[44]$} \\
\hline & Adult & & $\mathrm{V}$ & & & {$[46,47]$} \\
\hline TNC-CreER & $\mathrm{P} 1 \sim 3$ & & $\mathrm{~V}^{*}$ & & $\mathrm{SC}, \mathrm{BC}$ & [15] \\
\hline GABAa6-Cre & NA & $\mathrm{V}$ & & & & {$[35,36]$} \\
\hline Math1-Cre & NA & $\mathrm{V}$ & & & UBC & {$[37,38]$} \\
\hline GFAP-Cre & NA & $\mathrm{V}$ & $\mathrm{V}^{*}$ & & & {$[48]$} \\
\hline Pcp2/L7-Cre & NA & & & $\mathrm{V}$ & & {$[39,40]$} \\
\hline Shh-Cre & NA & & & $\mathrm{V}$ & & {$[15]$} \\
\hline
\end{tabular}

GCN, granule cell neuron; BG, Bergman glia; PC, Purkinje cell; IN, interneuron; *, cerebellar astrocyte; SC, stellate cells; BC, basket cell; UBC, Unipolar brush cells located in GCL; NA, not applicable.

for late-onset Cre expression in mature GCNs, Math1-CreER and NSE-CreERT2 are useful for targeting GCPs that give rise to GCNs in GCL $[35,38]$. However, Cre enzymes are also expressed in the non-cerebellar area in these mouse lines, such as the hippocampus, midbrain, medulla, spinal cord, and inner ear [27, 38, 42]. Unlike GCPs or GCNs, the Gli1 promoter is constitutively active in BGs. Thus, TAM administration around the weaning phase (P19 22) induces YFP expression in BGs. Several inducible Cre lines have been utilized for the study of gene function in BGs and specific types of astrocytes. While GFAP-CreER [21,43] and GLAST-CreER [44] induce recombination in BGs and astrocytes in a wide area of the brain, TNC-CreER can limit the reporter gene expression to the BGs and/or GABAergic interneurons in the cerebellum, depending on TAM administration [15].

\section{The perspective application of Gli1-CreERT2}

The cerebellum is the largest sensorimotor structure in the brain and has extensive connections with the brainstem and spinal cord. The cerebellum plays an important role in coordinating skilled voluntary movements by influencing muscle activity and controlling equilibrium and muscle tone through connections with the vestibular system and the spinal cord and its gamma motor neurons. Recently, there has been rapidly increasing evidence indicating the role of the cerebellum in emotion and cognition in addition to movement $[49,50]$. Long-term depression (LTD) is considered a cellular mechanism for cerebellar motor learning and is expressed as reduced responsiveness to transmitter glutamate $[51,52]$. In particular, PF-PC synapses are well-known sites for
LTD [53]. Intensive studies using Pcp2/L7-Cre have revealed that the molecular machinery, including $\mathrm{Ca}^{2+}$ influx, protein kinase $\mathrm{C}$, and endocytosis of AMPA-type glutamate receptors play critical roles in postsynaptic PCs [41]. By comparison, the presynaptic roles of PF in LTD induction are relatively unknown.

We show that Gli1-CreERT2 system can be applicable to conditional deletion of genes from cerebellar GCNs and/or BGs without altering gene expression in the non-cerebellar area and TAM treatment window of P4 7 allows excitatory GCN-specific deletion without affecting gene expression in GABAergic interneurons or PCs. We also propose that Gli1-CreERT2 can provide a tool to identify the molecular and cellular events in presynaptic PFs by allowing selective deletion of synaptic proteins with long half-lives in GCNs.

\section{ACKNOWLEDGEMENTS}

We thank Prof. Mi-Ryoung Song (Gwangju Institute of Science and Technology) and Prof. Yongsu Jeong (Kyung Hee University) for their informative and kind advice on mouse genetics. This research was supported by grants from the Korea Health Technology R\&D Project through the Korea Health Industry Development Institute (KHIDI), funded by the Ministry of Health \& Welfare (HI20C0457 to HS-K), the Ministry of Food and Drug Safety in 2021 (18172MFDS182-5 to HS-K), and the Basic Science Research Program through the National Research Foundation of Korea (NRF) funded by the Ministry of Education (NRF2020R1I1A1A01053066 to J-MC) of the Republic of Korea. 


\section{REFERENCES}

1. Kühn R, Schwenk F, Aguet M, Rajewsky K (1995) Inducible gene targeting in mice. Science 269:1427-1429.

2. Vaillant C, Monard D (2009) SHH pathway and cerebellar development. Cerebellum 8:291-301.

3. Ved N, Curran A, Ashcroft FM, Sparrow DB (2019) Tamoxifen administration in pregnant mice can be deleterious to both mother and embryo. Lab Anim 53:630-633.

4. Lizen B, Claus M, Jeannotte L, Rijli FM, Gofflot F (2015) Perinatal induction of Cre recombination with tamoxifen. Transgenic Res 24:1065-1077.

5. Carletti B, Rossi F (2008) Neurogenesis in the cerebellum. Neuroscientist 14:91-100.

6. Kitazawa S, Wolpert DM (2005) Rhythmicity, randomness and synchrony in climbing fiber signals. Trends Neurosci 28:611-619.

7. Wechsler-Reya RJ, Scott MP (1999) Control of neuronal precursor proliferation in the cerebellum by Sonic hedgehog. Neuron 22:103-114.

8. Fuccillo M, Joyner AL, Fishell G (2006) Morphogen to mitogen: the multiple roles of hedgehog signalling in vertebrate neural development. Nat Rev Neurosci 7:772-783.

9. De Luca A, Cerrato V, Fucà E, Parmigiani E, Buffo A, Leto K (2016) Sonic hedgehog patterning during cerebellar development. Cell Mol Life Sci 73:291-303.

10. Lewis PM, Gritli-Linde A, Smeyne R, Kottmann A, McMahon AP (2004) Sonic hedgehog signaling is required for expansion of granule neuron precursors and patterning of the mouse cerebellum. Dev Biol 270:393-410.

11. Wallace VA (1999) Purkinje-cell-derived Sonic hedgehog regulates granule neuron precursor cell proliferation in the developing mouse cerebellum. Curr Biol 9:445-448.

12. Dahmane N, Ruiz I Altaba A (1999) Sonic hedgehog regulates the growth and patterning of the cerebellum. Development 126:3089-3100.

13. Corrales JD, Rocco GL, Blaess S, Guo Q, Joyner AL (2004) Spatial pattern of Sonic hedgehog signaling through Gli genes during cerebellum development. Development 131:55815590 .

14. Ahn S, Joyner AL (2004) Dynamic changes in the response of cells to positive hedgehog signaling during mouse limb patterning. Cell 118:505-516.

15. Fleming JT, He W, Hao C, Ketova T, Pan FC, Wright CC, Litingtung Y, Chiang C (2013) The Purkinje neuron acts as a central regulator of spatially and functionally distinct cerebellar precursors. Dev Cell 27:278-292.
16. Ye L, Orynbayev M, Zhu X, Lim EY, Dereddi RR, Agarwal A, Bergles DE, Bhat MA, Paukert M (2020) Ethanol abolishes vigilance-dependent astroglia network activation in mice by inhibiting norepinephrine release. Nat Commun 11:6157.

17. Heo S, Diering GH, Na CH, Nirujogi RS, Bachman JL, Pandey A, Huganir RL (2018) Identification of long-lived synaptic proteins by proteomic analysis of synaptosome protein turnover. Proc Natl Acad Sci U S A 115:E3827-E3836.

18. Srinivas S, Watanabe T, Lin CS, William CM, Tanabe Y, Jessell TM, Costantini F (2001) Cre reporter strains produced by targeted insertion of EYFP and ECFP into the ROSA26 locus. BMC Dev Biol 1:4.

19. Choi CI, Yoon SP, Choi JM, Kim SS, Lee YD, Birnbaumer L, Suh-Kim H (2014) Simultaneous deletion of floxed genes mediated by CaMKIIa-Cre in the brain and in male germ cells: application to conditional and conventional disruption of Goa. Exp Mol Med 46:e93.

20. Choi JM, Kim SS, Choi CI, Cha HL, Oh HH, Ghil S, Lee YD, Birnbaumer L, Suh-Kim H (2016) Development of the main olfactory system and main olfactory epithelium-dependent male mating behavior are altered in Go-deficient mice. Proc Natl Acad Sci U S A 113:10974-10979.

21. Park YM, Chun H, Shin JI, Lee CJ (2018) Astrocyte specificity and coverage of hGFAP-CreERT2 [Tg(GFAP-Cre/ ERT2)13Kdmc] mouse line in various brain regions. Exp Neurobiol 27:508-525

22. Behesti H, Marino S (2009) Cerebellar granule cells: insights into proliferation, differentiation, and role in medulloblastoma pathogenesis. Int J Biochem Cell Biol 41:435-445.

23. Consalez GG, Goldowitz D, Casoni F, Hawkes R (2021) Origins, development, and compartmentation of the granule cells of the cerebellum. Front Neural Circuits 14:611841.

24. Corrales JD, Blaess S, Mahoney EM, Joyner AL (2006) The level of Sonic hedgehog signaling regulates the complexity of cerebellar foliation. Development 133:1811-1821.

25. Cheng FY, Fleming JT, Chiang C (2018) Bergmann glial Sonic hedgehog signaling activity is required for proper cerebellar cortical expansion and architecture. Dev Biol 440:152-166.

26. Sotelo C (2004) Cellular and genetic regulation of the development of the cerebellar system. Prog Neurobiol 72:295-339.

27. Machold R, Fishell G (2005) Math1 is expressed in temporally discrete pools of cerebellar rhombic-lip neural progenitors. Neuron 48:17-24.

28. Consalez GG, Hawkes R (2013) The compartmental restriction of cerebellar interneurons. Front Neural Circuits 6:123.

29. Leto K, Carletti B, Williams IM, Magrassi L, Rossi F (2006) Different types of cerebellar GABAergic interneurons origi- 
nate from a common pool of multipotent progenitor cells. J Neurosci 26:11682-11694.

30. Leto K, Rolando C, Rossi F (2012) The genesis of cerebellar GABAergic neurons: fate potential and specification mechanisms. Front Neuroanat 6:6.

31. Huang X, Liu J, Ketova T, Fleming JT, Grover VK, Cooper MK, Litingtung Y, Chiang C (2010) Transventricular delivery of Sonic hedgehog is essential to cerebellar ventricular zone development. Proc Natl Acad Sci U S A 107:8422-8427.

32. Zhang L, Goldman JE (1996) Generation of cerebellar interneurons from dividing progenitors in white matter. Neuron 16:47-54.

33. Hoshino M, Nakamura S, Mori K, Kawauchi T, Terao M, Nishimura YV, Fukuda A, Fuse T, Matsuo N, Sone M, Watanabe M, Bito H, Terashima T, Wright CV, Kawaguchi Y, Nakao K, Nabeshima Y (2005) Ptfla, a bHLH transcriptional gene, defines GABAergic neuronal fates in cerebellum. Neuron 47:201-213.

34. Weisheit G, Gliem M, Endl E, Pfeffer PL, Busslinger M, Schilling K (2006) Postnatal development of the murine cerebellar cortex: formation and early dispersal of basket, stellate and Golgi neurons. Eur J Neurosci 24:466-478.

35. Fünfschilling U, Reichardt LF (2002) Cre-mediated recombination in rhombic lip derivatives. Genesis 33:160-169.

36. Aller MI, Jones A, Merlo D, Paterlini M, Meyer AH, Amtmann U, Brickley S, Jolin HE, McKenzie AN, Monyer H, Farrant M, Wisden W (2003) Cerebellar granule cell Cre recombinase expression. Genesis 36:97-103.

37. Kim E, Wang Y, Kim SJ, Bornhorst M, Jecrois ES, Anthony TE, Wang C, Li YE, Guan JL, Murphy GG, Zhu Y (2014) Transient inhibition of the ERK pathway prevents cerebellar developmental defects and improves long-term motor functions in murine models of neurofibromatosis type 1. Elife 3:e05151.

38. Men Y, Zhang A, Li H, Jin Y, Sun X, Li H, Gao J (2015) LKB1 regulates cerebellar development by controlling sonic hedgehog-mediated granule cell precursor proliferation and granule cell migration. Sci Rep 5:16232.

39. Barski JJ, Dethleffsen K, Meyer M (2000) Cre recombinase expression in cerebellar Purkinje cells. Genesis 28:93-98.

40. Driver AM, Shumrick C, Stottmann RW (2017) Ttc21b is required in bergmann glia for proper granule cell radial migration. J Dev Biol 5:18.

41. Sługocka A, Wiaderkiewicz J, Barski JJ (2017) Genetic targeting in cerebellar purkinje cells: an update. Cerebellum 16:191-
202.

42. Pohlkamp T, Steller L, May P, Günther T, Schüle R, Frotscher M, Herz J, Bock HH (2014) Generation and characterization of an Nse-CreERT2 transgenic line suitable for inducible gene manipulation in cerebellar granule cells. PLoS One 9:e100384.

43. Chow LM, Zhang J, Baker SJ (2008) Inducible Cre recombinase activity in mouse mature astrocytes and adult neural precursor cells. Transgenic Res 17:919-928.

44. Parmigiani E, Leto K, Rolando C, Figueres-Oñate M, LópezMascaraque L, Buffo A, Rossi F (2015) Heterogeneity and bipotency of astroglial-like cerebellar progenitors along the interneuron and glial lineages. J Neurosci 35:7388-7402.

45. Guo Z, Wang X, Xiao J, Wang Y, Lu H, Teng J, Wang W (2013) Early postnatal GFAP-expressing cells produce multilineage progeny in cerebrum and astrocytes in cerebellum of adult mice. Brain Res 1532:14-20.

46. Mori T, Tanaka K, Buffo A, Wurst W, Kühn R, Götz M (2006) Inducible gene deletion in astroglia and radial glia--a valuable tool for functional and lineage analysis. Glia 54:21-34.

47. Zhang RS, Liakath-Ali K, Südhof TC (2020) Latrophilin-2 and latrophilin-3 are redundantly essential for parallel-fiber synapse function in cerebellum. Elife 9:e54443.

48. Yang H, Zhu Q, Cheng J, Wu Y, Fan M, Zhang J, Wu H (2019) Opposite regulation of $\mathrm{Wnt} / \beta$-catenin and Shh signaling pathways by Rack1 controls mammalian cerebellar development. Proc Natl Acad Sci U S A 116:4661-4670.

49. Koziol LF, Budding D, Andreasen N, D'Arrigo S, Bulgheroni S, Imamizu H, Ito M, Manto M, Marvel C, Parker K, Pezzulo G, Ramnani N, Riva D, Schmahmann J, Vandervert L, Yamazaki T (2014) Consensus paper: the cerebellum's role in movement and cognition. Cerebellum 13:151-177.

50. Lawrenson C, Bares M, Kamondi A, Kovács A, Lumb B, Apps R, Filip P, Manto M (2018) The mystery of the cerebellum: clues from experimental and clinical observations. Cerebellum Ataxias 5:8.

51. Massey PV, Bashir ZI (2007) Long-term depression: multiple forms and implications for brain function. Trends Neurosci 30:176-184.

52. Hirano T (2013) Long-term depression and other synaptic plasticity in the cerebellum. Proc Jpn Acad Ser B Phys Biol Sci 89:183-195.

53. Hoxha E, Tempia F, Lippiello P, Miniaci MC (2016) Modulation, plasticity and pathophysiology of the parallel fiberpurkinje cell synapse. Front Synaptic Neurosci 8:35. 